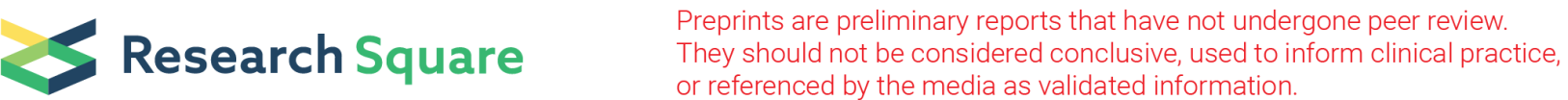

\section{Performance and potential clinical impact of D 20 - labeled single-cell Raman spectroscopy for direct antimicrobial susceptibility testing of Klebsiella pneumoniae}

\section{Dong-yu Liang}

Jiading District Central Hospital Affiliated Shanghai University of Medicine \& Health Sciences

\section{Lijuan Shen}

Jiading District Central Hospital Affiliated Shanghai University of Medicine \& Health Sciences

\section{Fei Wang}

Jiading District Central Hospital Affiliated Shanghai University of Medicine \& Health Sciences

\section{Yizhi Song}

Chinese Academy of Sciences

Qing Chang ( $\sim$ jd_changq@sumhs.edu.cn )

Jiading District Central Hospital Affiliated Shanghai University of Medicine \& Health Sciences

\section{Research Article}

Keywords: Single-cell Raman spectroscopy, D2O-labeled, Antimicrobial susceptibility, Klebsiella pneumoniae

Posted Date: March 1st, 2022

DOI: https://doi.org/10.21203/rs.3.rs-1304498/v1

License: (c) (i) This work is licensed under a Creative Commons Attribution 4.0 International License.

Read Full License 


\section{Abstract}

Rapid and accurate antimicrobial susceptibility testing (AST) of bacterial infections can improve patients' outcome. In this study, 29 Klebsiella pneumoniae strains isolated from sputum specimens from ICU patients at the Jiading Central Hospital were compared using $\mathrm{D}_{2} \mathrm{O}$-Raman AST and traditional AST (Vitek 2). The analysis time was $4-4.5 \mathrm{~h}$ for a complete $\mathrm{D}_{2} \mathrm{O}$-Raman $\mathrm{AST}$ result. Compared with the Vitek 2 , the results of $\mathrm{D}_{2} \mathrm{O}$-Raman spectroscopy showed good agreement for susceptibility and resistance $(\mathrm{S} / \mathrm{R})$, with a concordance of $89 \%$. Most discrepant results were observed for intermediate and susceptible categories, as $10 \%$ of isolates labeled as intermediate and susceptible by Vitek 2 were almost all categorized as resistant by $\mathrm{D}_{2} \mathrm{O}$-Raman spectroscopy. Moreover, discriminant factor analysis of our $\mathrm{D}_{2} \mathrm{O}$ Raman results led to successfully discriminating the type of resistance. In addition, we evaluated the behavior of clinicians in adjusting antibiotic therapies according to routine AST results. For 9 out of 10 patients, antibiotic therapy was adjusted after the reporting of AST results. With some improvements, $\mathrm{D}_{2} \mathrm{O}$-Raman spectroscopy provides an accurate and rapid preliminary AST result and may have at least a potential clinical benefit for patients.

\section{Introduction}

Bacterial infection is one of the major causes of human disease, and antibiotics have been developed for killing bacterial pathogens[1]. However, the number of bacteria resistant to broad-spectrum antibiotics is increasing because of antibiotic misuse and abuse, posing a great threat to human health[2]. Rapid and accurate characterization of bacterial susceptibility to antibiotics is therefore urgently needed for guiding correct and effective antibiotic therapy in patients with serious infections.

Klebsiella pneumoniae, a Gram-negative bacterium, is one of the most important causes of nosocomial infections[3]. Klebsiella pneumoniae is also the most common multi-drug-resistant bacteria[4]. This bacterium can cause pneumonia, urinary system infection, peritonitis, sepsis, and other diseases in patients[5]. Especially for patients in the ICU ward, due to the serious disease of these patients, the immune system is often compromised. If a patient infected with Klebsiella pneumoniae does not receive effective treatment, it can affect the patient's life[6]. Thus, the faster information on bacterial susceptibility to antibiotics is available, the faster the patients' treatment can be optimized, minimizing the risk of developing antibiotic resistance as well as use of costly pharmaceuticals. This potentially saves the patients' life and reduces their irreversible lone-term side effects.

Heavy water $\left(\mathrm{D}_{2} \mathrm{O}\right)$-based single-cell Raman micro-spectroscopy ( $\mathrm{D}_{2} \mathrm{O}$-Raman) combining Raman microspectroscopy with heavy water $\left(D_{2} 0\right)$ has been demonstrated for quantitative assessment of the metabolism-inhibiting effects of drugs[7]. Raman shift at the C-D (carbon-deuterium vibration) band around 2040-2300 $\mathrm{cm}^{-1}$, which has been considered a universal biomarker for general metabolic activity, has been used to probe bacterial responses to different drugs by simple adding heavy water $\left(D_{2} O\right)$ to samples[8]. In addition, based on the metabolic activity, the minimum inhibitory concentration 
(MIC-MA) has been proposed for evaluating the metabolism-inhibiting efficacy of drugs. Furthermore, this method is able to detect "nongrowing but metabolically active" (NGMA) cells, which can lead to many latent or recurring infections[9]. Despite this progress, $\mathrm{D}_{2} \mathrm{O}$-Raman spectroscopy has not been established as a reliable and rapid AST tool for clinical applications. Here, we tested 29 Klebsiella pneumoniae isolates from ICU patients with antibiotics resistance in conditions as close as possible to a real clinic using commercially available antibiotics at the same concentration and preparation as those used in clinical treatments. The results showed that, compared with the Vitek 2, the results of $\mathrm{D}_{2} \mathrm{O}$-Raman spectroscopy showed good agreement in terms of susceptibility and resistance $(S / R)$, with a concordance of $90 \%$. Moreover, discriminant factor analysis (DFA) of these $\mathrm{D}_{2} \mathrm{O}$-Raman results were successful for discriminating the type of resistance. These promising results highlight the potential of this technique for faster determination of bacterial susceptibility to certain antibiotics.

\section{Materials And Methods}

\section{Bacterial samples and preparation}

The 29 Klebsiella pneumoniae strains used in this study were all isolated from sputum specimens from ICU patients. Prior to $\mathrm{D}_{2} \mathrm{O}$-Raman investigation, their antimicrobial susceptibility was detected by Vitek2/Disk diffusion. The antimicrobial susceptibility profiles for a range of antibiotics are summarized in Table 1.

Each bacterial strain was grown in $\mathrm{MH}$ medium to an optical density $\left(\mathrm{OD}_{600}\right)$ of 0.5 . The bacterial concentration at this OD was diluted 1:200 using MH medium, then $5 \mu \mathrm{l}$ of this solution was added to preprepared antibiotics sensitivity cultures at $35^{\circ} \mathrm{C}$ with $150 \mathrm{rpm}$ shaking for $2 \mathrm{~h}$. After incubation with $66 \mu \mathrm{l}$ of heavy water for another $1 \mathrm{~h}$, the bacterial solution was centrifuged and washed with sterile water three times. After the last time, the supernatant was aspirated, and $50 \mu \mathrm{L}$ of sterilized deionized water was added to the pellet, and $2.5 \mu \mathrm{L}$ of each sample was dropped on the chip for Raman testing.

\section{Acquisition and analysis of single-cell Raman data}

For quantification of the extent of deuterium incorporation, C-D ratio, which was defined as the percentage of the integrated spectral intensity of the C-D band, was calculated by comparing the sum of the $C-D$ band with the predominant $C-H$ band $\left(2800-3100 \mathrm{~cm}^{-1}\right)$. MICs were determined when the C-D ratio of the samples with antibiotics showed no significant difference relative to that of a negative control using Tukey's HSD test $(p<0.01)$.

\section{Patient clinical information}

For each patient included in this study, medical records, the empiric antibiotic therapy, and antibiotic therapy after AST results were retrospectively assessed. The date and time of every modification in antibiotic therapy was collected and compared. The effect of AST results on the antibiotic therapy of each patient was retrospectively discussed with a panel of microbiologists and clinicians. 


\section{Statistical analysis}

All data were analyzed in $\mathrm{R}$ (version 3.4.3). Statistical comparisons were performed using Wilcox test, and $p<0.05$ was considered significant. Principal component analysis (PCA) and DFA were used to distinguish antibiotic susceptibility.

\section{Results}

\section{Tracking deuterium incorporation in Klebsiella pneumoniae via single-cell Raman spectroscopy}

Klebsiella pneumoniae is one of the most important causes of nosocomial infections. Here, we tested the general applicability of the $\mathrm{D}_{2} \mathrm{O}$-Raman method for assessing bacterial metabolic activity. We started by probing the sensitivity of bacterial growth to the $\mathrm{D}_{2} \mathrm{O}$ level in culture medium. For this, a broad C-D Raman band at 2040-2300 $\mathrm{cm}^{-1}$ emerged, which followed by a Raman shift of the original C-H peak at 2800$3100 \mathrm{~cm}^{-1}$. No visible C-D band was observed in $\mathrm{D}_{2} \mathrm{O}$-free cultures (Fig. 1). The batch calculations of C-D ratios $(C D /(C D+C H))$ were used to determine $S / R$.

\section{Establishing $\mathrm{D}_{2} \mathrm{O}$-Raman as a rapid and accurate method for AST}

One of the key issues in applying $\mathrm{D}_{2} \mathrm{O}$-Raman for rapid AST is its consistency with growth-based S/R clinical interpretive criteria. To seek an appropriate antibiotic treatment by $\mathrm{D}_{2} \mathrm{O}$-Raman, ensuring a rapid and reliable AST, three susceptible and three resistant Klebsiella pneumoniae strains that were previously confirmed by Vitek2 and disk diffusion, were incubated with ampicillin (Amp), gentamicin (GM), and levofloxacin (LEV), respectively, in $\mathrm{D}_{2} \mathrm{O}$ media for different times. These antibiotics have different mechanisms against bacteria. Amp and GM act against bacteria through inhibiting the synthesis of bacterial cell wall components and proteins, respectively[10,11]. LEV is a bacteriostatic, which acts by preventing bacterial DNA synthesis and replication[12]. At an incubation time of $1 \mathrm{~h}$, with increasing antibiotic doses, the C-D ratios of susceptible Klebsiella pneumoniae in response to all three antibiotics decreased (Figs. 2B and $C$ ), while that of three resistant strains remained unchanged. From the corresponding C-D Raman bands (Fig. 2A), a susceptible strain could not be seen while a resistant strain still displayed a clear C-D band.

\section{Comparison of $\mathrm{D}_{2} \mathrm{O}-\mathrm{Raman}$ AST and Vitek 2}

For antibiotic susceptibility testing, traditional methods are mostly based on MIC, which is defined as the minimal dose under which no visible bacterial growth can be detected. The MICs of 10 Klebsiella pneumoniae isolates were assessed by both the Vitek 2 Automated Microbiology System and $\mathrm{D}_{2} \mathrm{O}$ Raman spectroscopy. 
In total, five samples showed total agreement in their results compared with the Vitek 2 AST system (Table 2). Except for one sample that had five discordant antibiotics, the other samples had only one or two discrepancies. The average concordance rate was $89 \%$ between the two methods. The major discrepancy was shown most in the intermediate and susceptible categories, as $10 \%$ of isolates labeled as susceptible or intermediate by Vitek 2 were all categorized as resistant by $\mathrm{D}_{2} \mathrm{O}$-Raman spectroscopy.

Under the microscope, these discrepant isolates could be observed to have a small amount of normal or elongated bacteria (Fig. 3). Raman detection found that these bacteria had deuterium peaks, indicating that, although the concentration of antibiotics inhibited the proliferation of these bacteria, the remaining bacteria still had strong metabolic activity, indicative of NGMA cells.

\section{Discrimination of 13 Klebsiella pneumoniae isolates}

According to the results of our antibiotic susceptibility testing, another 13 Klebsiella pneumoniae isolates were divided into antibiotic-resistant (R) (containing 5 isolates), antibiotic-intermediate (I) (containing 3 isolates) and antibiotic-susceptible (S) (containing 3 isolates), based on AST results from Vitek2/disk diffusion. Differences in intensities were not clearly visible between these 13 strains (Fig. 4A). Next, PCA and DFA were performed. The score plots of PCA and DFA illustrated that the antibiotic susceptibility could only be distinguished by DFA (Figs. 4B and C). Moreover, these results suggest that Raman spectroscopy has the ability to discriminate and predict antibiotic susceptibility, even though these strains were genetically very close.

\section{Clinical data}

Clinical data regarding the antibiotic therapy in 10 different patients are summarized in Table 3. For nine patients, the clinician was influenced by the AST result to adjust the therapy used. Of all patients that were switched based on AST results, the antibiotic therapy was de-escalated in $68 \%$ of these cases $(6 / 9)$. The reason that the AST result did not have an impact on the antibiotic therapy was based on the resistance profile of the microorganisms observed or due to other patient characteristics. For one sample, the reason for not adjusting the antibiotic therapy was that the empirical antibiotic therapy was effective. Although the AST results had a significant impact on the antibiotic therapy used, there were still some inconsistencies. For the 14th, 28th, and 39th samples, the AST results from Vitek 2 categorized the strains as susceptibility or intermediate susceptibility to Ceftazidime, Piperacillin, and Imipenem, respectively. Unfortunately, these antibiotic therapies were not effective. However, all these were categorized as resistant by $\mathrm{D}_{2} \mathrm{O}$-Raman spectroscopy. The reason may be NGMA cells or others unknown factors.

\section{Discussion}

In recent years, $\mathrm{D}_{2} \mathrm{O}$-Raman methods have been reported to be used for identification of antibiotic resistance through measuring the cellular metabolic activity in response to antibiotic treatment at the single-cell level[13]. In principle, compared with other stable isotopes such as $C 13, D_{2} O$ uptake is more 
sensitive and rapid[14]. Moreover, dead cells are unable to form C-D bands in the presence of $\mathrm{D}_{2} \mathrm{O}$, which allows researchers to distinguish them from NGMA cells[15]. Previous studies have focused on its rapid abilities for monitoring phenotypic responses by bacteria in response to antibiotic treatment[16].

This study provides strain-specific identification at clinically relevant infection levels. Twenty-nine Klebsiella pneumoniae isolates from sputum samples of ICU patients were included in this study. In a comparison of the results from the Vitek2 Automated Microbiology System and Raman-D2O spectroscopy for interpretation of antibiotic susceptibility, most of the antibiotics had moderate to perfect agreement between these two methods, with a coincidence rate of about $89 \%$. The major discrepancy was in the intermediate and susceptible categories, as $10 \%$ of isolates labeled as intermediate or susceptibility by Vitek 2 were all categorized as resistant by $\mathrm{D}_{2} \mathrm{O}$-Raman spectroscopy. From a clinical standpoint, reclassifying antibiotic susceptibility from intermediate or susceptibility to resistant is unlikely to adversely affect a patient, as it simple removes it from being a therapeutic consideration.

Moreover, we demonstrated the use of Raman spectral information in identifying bacterial cell type. Interestingly, the 13 Klebsiella pneumoniae isolates could be well distinguished by DFA. The accuracy of the predictions here demonstrates the ability of Raman spectroscopy to produce fine grain, reliable, phenotypic signatures for the characterization of antibiotic resistance even if strains are closely related.

In order to assess the potential clinical impact of our $\mathrm{D}_{2} \mathrm{O}$ assay, we evaluated the behavior of clinicians in their prescribed adjusting antibiotic therapies based on AST results. We showed that the AST result from our $\mathrm{D}_{2} \mathrm{O}$ assay had greater potential impact for optimizing antibiotic therapies. We intend to continue our research with an enlarged database, and to expand this study to include different types of bacteria, leading to a method that offers the potential for clinical implementation.

\section{Declarations}

\section{Acknowledgements}

We thank LetPub (www.letpub.com) for its linguistic assistance during the preparation of this manuscript.

\section{Authors' contributions}

Liang Dongyu assisted with data analysis, interpretation, and drafting of the manuscript. Shen Lijuan and Wang Fei contributed to collecting and measuring samples in this study. Song Yizhi contributed to data interpretation and critically reviewed the manuscript. Chang Qing assisted with study design, statistical analysis. All authors planned the study design, contributed to the interpretation of the data, drafted and approved the submitted manuscript.

\section{Funding}


This study was supported by the Natural Science Foundation of Shanghai, China (Grant number:19ZR1444800).

\section{Availability of data and materials}

All data generated or analysed during this study are included in this published article]

\section{Ethics approval and consent to participate}

The experimental protocol was established, according to the ethical guidelines of the Helsinki Declaration and was approved by the Human Ethics Committee of Jiading District Central Hospital . Written informed consent was obtained from individual or guardian participants.

\section{Consent for publication}

Not applicable.

\section{Competing interests}

The authors declare no conflicts of interest.

\section{References}

1. Ahmed I, Sajed M, Sultan A, et al. The erratic antibiotic susceptibility patterns of bacterial pathogens causing urinary tract infections[J]. Excli Journal, 2015, 14:916-925.

2. Bush K. Antibacterial drug discovery in the 21 st century[J]. Clinical Microbiology and Infection, 2004, 10(Supplement s4):10-17.

3. Effah C Y, Sun T, Liu S, et al. Klebsiella pneumoniae: An increasing threat to public health[J]. Annals of Clinical Microbiology and Antimicrobials, 2020, 19(1).

4. Zhang $\mathrm{P}$, Shi Q, Hu H, et al. Emergence of ceftazidime/avibactam resistance in carbapenem-resistant Klebsiella pneumoniae in China[J]. Clinical Microbiology and Infection, 2019, 26(1).

5. M Cátia, Juliana $M$, Adriana $B$, et al. Klebsiella pneumoniae causing urinary tract infections in companion animals and humans: population structure, antimicrobial resistance and virulence genes[J]. Journal of Antimicrobial Chemotherapy, 2018(3):3.

6. Xiaohua Q, Shi W, Min H, et al. The Colonization of Carbapenem-Resistant Klebsiella pneumoniae: Epidemiology, Resistance Mechanisms, and Risk Factors in Patients Admitted to Intensive Care Units in China[J]. The Journal of Infectious Diseases(Supplement_2):Supplement_2.

7. A sensitive and rapid bacterial antibiotic susceptibility test method by surface enhanced Raman spectroscopy[J]. Brazilian Journal of Microbiology, 2020, 51(3):875-881.

8. Berry D, Mader E, Lee T K, et al. Tracking heavy water (D 20 ) incorporation for identifying and sorting active microbial cells[J]. Proceedings of the National Academy of Sciences, 2014, 112(2). 
9. Tao Y, Wang Y, Huang S, et al. Metabolic-Activity-Based Assessment of Antimicrobial Effects by D2OLabeled Single-Cell Raman Microspectroscopy[J]. Analytical Chemistry, 2017, 89(7):4108-4115.

10. Bolivar F. Construction and characterization of new cloning vehicles. I. Ampicillin-resistant derivatives of the plasmid pMB9.[J]. Gene, 1977, 2.

11. Appel G B, Neu H C. Gentamicin in 1978[J]. Annals of Internal Medicine, 1978, 89(4):528.

12. Izadi M, Dadsetan B, Najafi Z, et al. Levofloxacin Versus Ceftriaxone and Azithromycin Combination in the Treatment of Community Acquired Pneumonia in Hospitalized Patients[J]. Recent Patents on Anti-Infective Drug Discovery, 2018, 13(3).

13. Carey $P$ R, Heidari-Torkabadi $\mathrm{H}$. New techniques in antibiotic discovery and resistance: Raman spectroscopy[J]. Annals of the New York Academy of Sciences, 2015, 1354(1):67-81.

14. Song Y, Kaster A K, Vollmers J, et al. Single-cell genomics based on Raman sorting reveals novel carotenoid-containing bacteria in the Red Sea[J]. Microbial Biotechnology, 2017, 10(1):125-137.

15. Harrison J P, David B. Vibrational Spectroscopy for Imaging Single Microbial Cells in Complex Biological Samples[J]. Frontiers in Microbiology, 2017, 8:675.

16. Maurer F P, Christner M, Hentschke M, et al. Advances in Rapid Identification and Susceptibility Testing of Bacteria in the Clinical Microbiology Laboratory: Implications for Patient Care and Antimicrobial Stewardship Programs.[J]. Infectious Disease Reports, 2017, 9(1).

\section{Tables}

Table 1 to 3 is available in the Supplemental Files section.

\section{Figures}




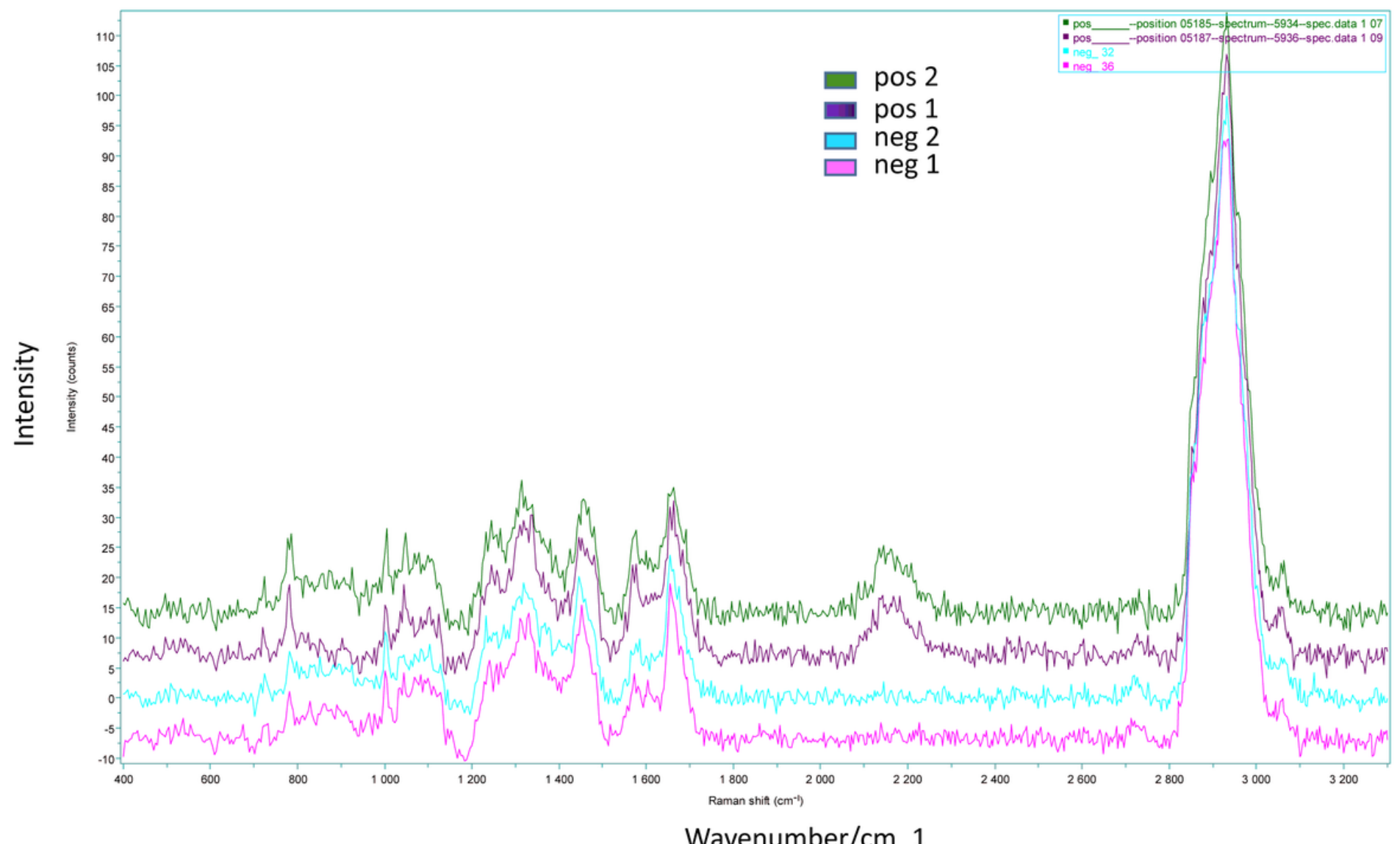

Figure 1

Figure 1

$\mathrm{D}_{2} \mathrm{O}$-Raman spectra of cells incubated with $\mathrm{MH}$ culture medium treated with heavy water without antibiotic treatment. 

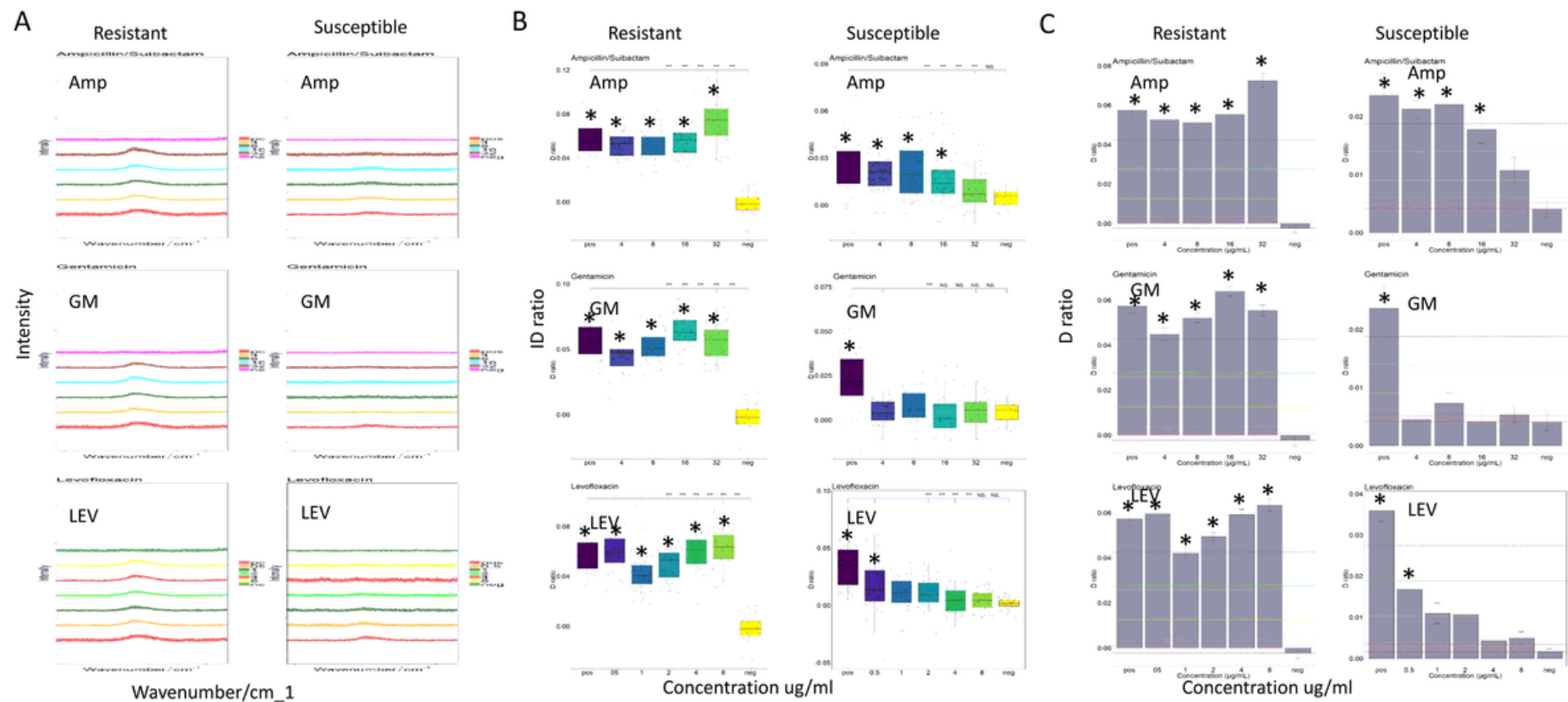

Figure 2

Figure 2

$\mathrm{D}_{2} \mathrm{O}$-Raman spectroscopy detects susceptible and resistant Klebsiella pneumoniae strains. (A) C-D bands of susceptible and resistant strains after 1-h incubation with different concentration antibiotics. (B, C) C-D ratios of susceptible and resistant strains after 1-h incubation with different concentrations of antibiotics. (Wilcox test, compared with a negative control, ${ }^{*} \mathrm{P}<0.05$, NS. not significant.)

A

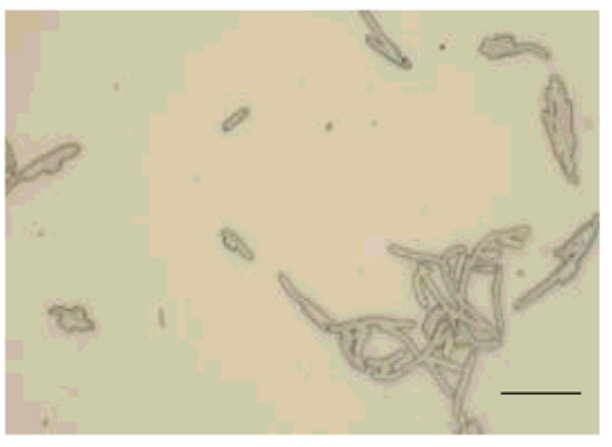

B

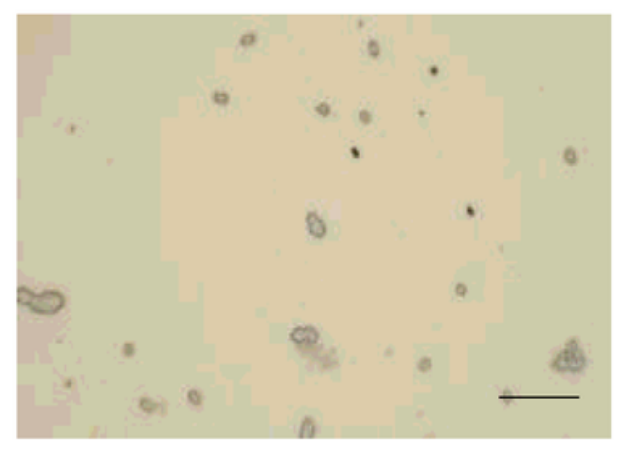

C

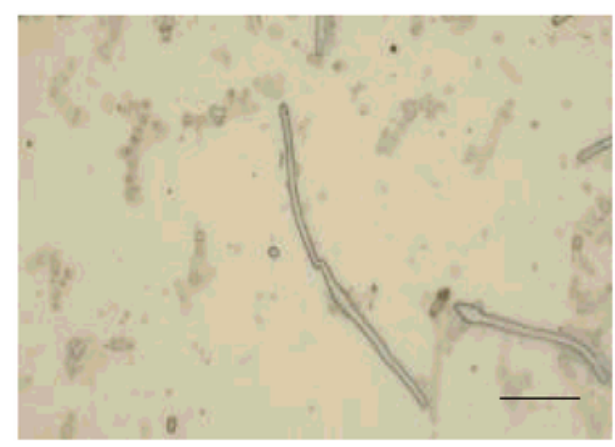

Figure 3

Figure 3

The morphology of Klebsiella pneumoniae collected by Raman-microscopy. (A) Normal isolate. (B) Susceptible isolate. (C) Resistant isolate. Scale bar: $10 \mu \mathrm{m}$. 


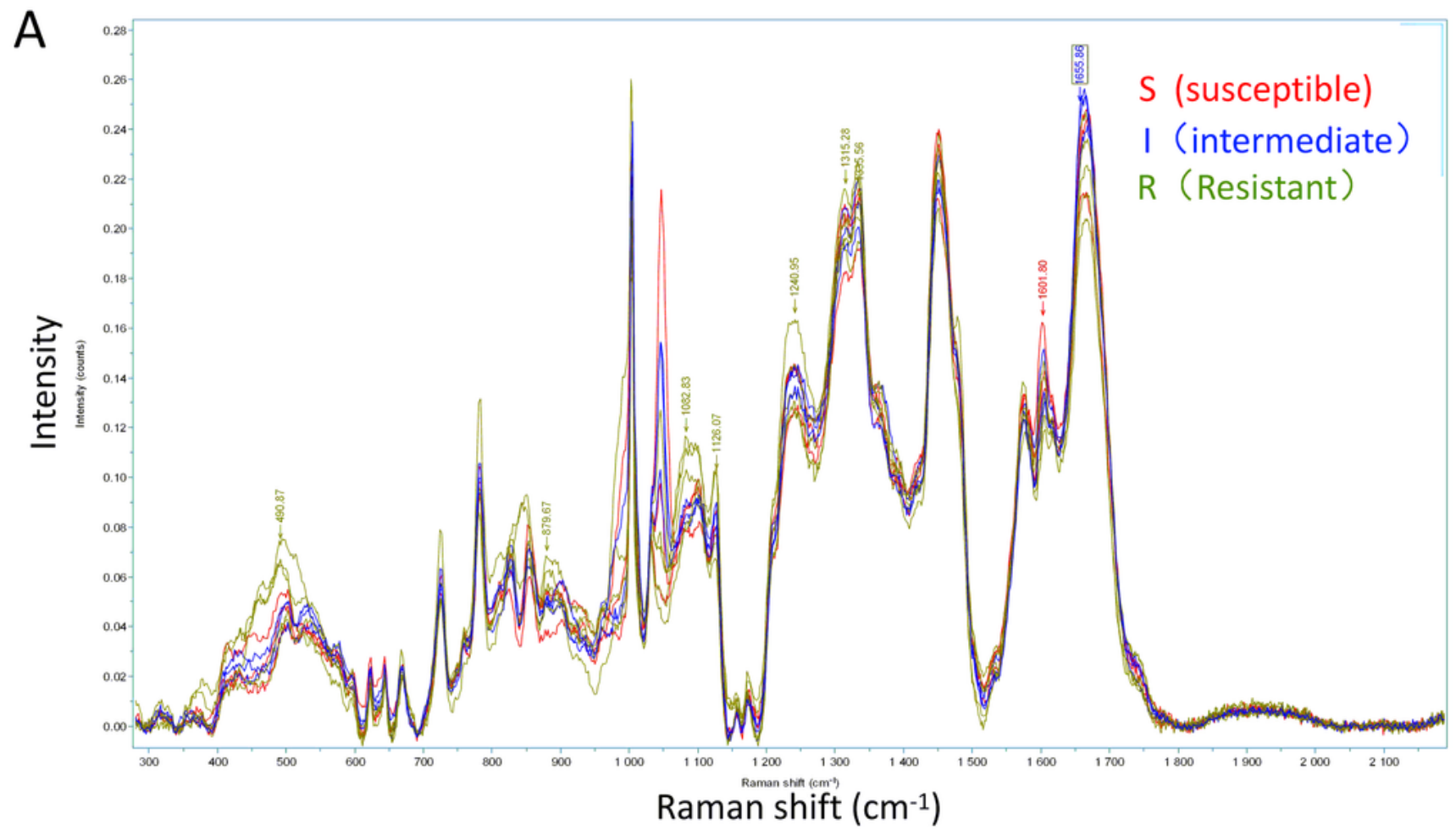

B

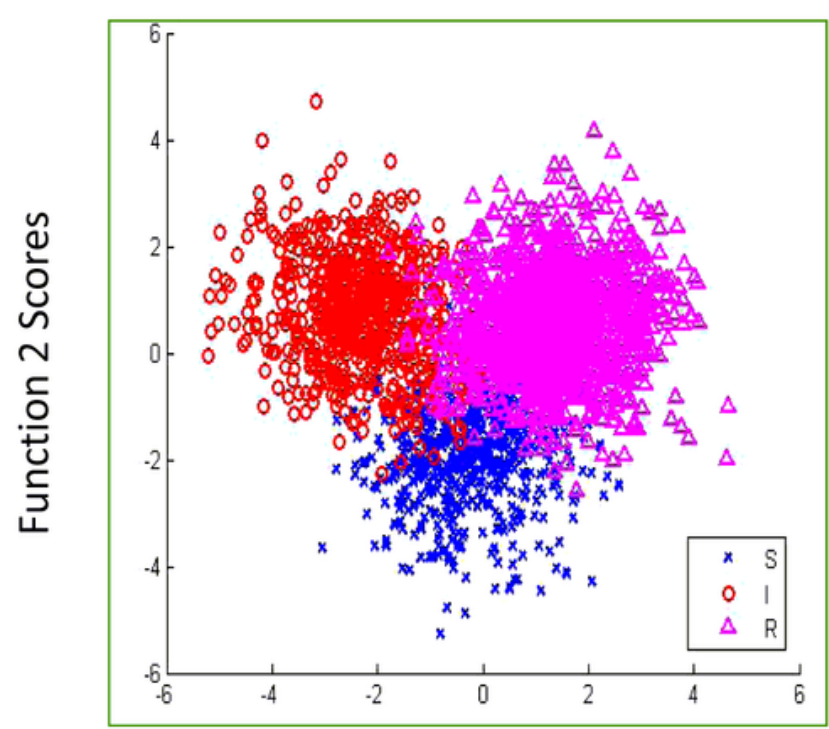

Function 1 Scores
$\mathrm{C}$

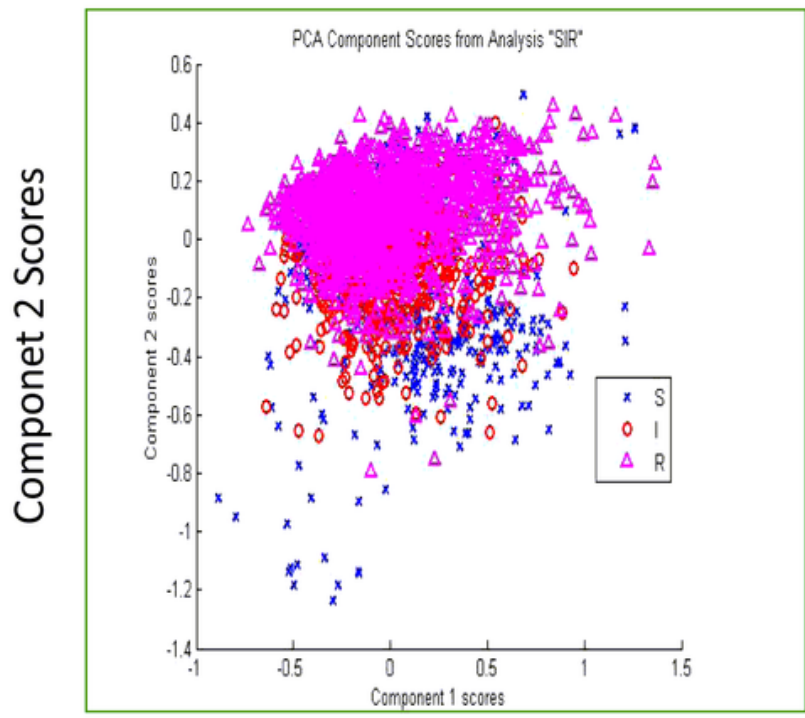

Componet 1 Scores

Figure 4

Figure 4

Label-free measurement of 13 Klebsiella pneumoniae isolates and discrimination of strains. (A) Raman spectra of 13 Klebsiella pneumonia isolates. (B) Discrimination of strains by discriminant factor analysis (DFA). (C) Discrimination of strains by principal component analysis (PCA).

\section{Supplementary Files}


This is a list of supplementary files associated with this preprint. Click to download.

- table1.tif

- table2.tif

- table3.tif 